\title{
Outcome of patients admitted during off hours in Moroccan intensive care unit
}

This article was published in the following Dove Press journal:

International Journal of General Medicine

27 February 2014

Number of times this article has been viewed

\author{
Mina Elkhayari' \\ Othmane Dilai \\ Amra Ziadi ${ }^{2}$ \\ Abdelhamid Hachimi' \\ Mohammed Abdenasser \\ Samkaoui ${ }^{2}$ \\ 'Medical Intensive Care Unit, \\ Mohammed VI University Hospital, \\ Faculty of Medicine and Pharmacy, \\ University Cadi Ayyad, Marrakech, \\ Morocco; ${ }^{2}$ Surgical Intensive Care \\ Unit, Mohammed VI University \\ Hospital, Faculty of Medicine and \\ Pharmacy, University Cadi Ayyad, \\ Marrakech, Morocco
}

Background: The first few hours after intensive care unit (ICU) admission, where a patient's condition is stabilized and treatment plans are formulated, are crucial to patient outcome. Although admission of patients who are unstable to ICU occurs 24 hours a day, not all units maintain the same level of staffing during off hours. We evaluated whether ICU admission during off hours affects mortality in a Moroccan ICU with the same level of staffing.

Methods: This prospective study was carried out at an ICU in a Moroccan hospital during 6 months. Demographic, clinic, acute physiology and chronic health evaluation score, length of stay, time of admission (day time or off hours), and ICU mortality data were collected. The mortality in the ICU was the end point of the study. Logistic regression analysis was used to identify risk factors associated with ICU mortality at various day and time of admission.

Results: A total of 195 patients were included in the study; 125 (63.6\%) were admitted during the day time and 70 (36.4\%) were admitted during off hours. Most of the patients admitted during the off hours were male ( $75 \%$ versus $58 \%$ during the day time, $P=0.01)$. Patients admitted in off hours after traumatism were more frequent than those admitted during the day time ( $64 \%$ versus $24 \%, P=0.001$ ). There was no significant difference in ICU mortality for time of ICU admission $(P=0.05)$.

Conclusion: We can conclude that off hours care is not necessarily inadequate. For ICU managers, it is important to know how to maintain adequate quality of care around the clock.

Keywords: intensive care unit, day time, off hours, mortality

\section{Background}

Patients in the intensive care unit (ICU) often have several organ abnormalities and physiological disarrangements that require highly complex and timely diagnostic tests and therapies. ${ }^{1}$ The first few hours after ICU admission, where a patient's condition is stabilized and treatment plans are formulated, are crucial to patient outcome. ${ }^{2}$ Ideally, the critical care unit has to be organized in such a way that optimal treatment is available to all patients day and night. However, the availability and quality of personnel and technology are often different during day time hours as compared with off hours. Several authors have studied the difference in outcome between patients admitted during day time hours as compared with off hours in ICU and the results were contradictory. ${ }^{3-5}$ The current study was undertaken to investigate the demographic characteristics and outcomes of patients admitted in ICU during off hours in a Moroccan tertiary hospital with uniform staffing and ready access to diagnostic and therapeutic options.
Correspondence: Mina Elkhayari Medical Intensive Care Unit, Mohammed VI University Hospital, Faculty of Medicine and Pharmacy, University Cadi Ayyad, 2D Nakhil3, Gueliz, Marrakech, 40000, Morocco Tel +212661276768

Email amina.elkhayari@gmail.com 


\section{Methods}

This prospective cohort study was carried out in a Moroccan ICU. The consent and approval of the study was made by the local ethics community. All patients admitted to the ICU between April 1 and September 30, 2012, were included. Patients were excluded if they were younger than 16 years of age or if they were admitted for less than 8 hours. Only the first ICU admission was taken into account for patients who were readmitted to the ICU. Mortality in the ICU was the end point of the study. Day time hours were defined as the hours between 8 am and 8 pm on weekdays and between 9 am and $6 \mathrm{pm}$ during the weekend. Off hours were defined as the hours between $8 \mathrm{pm}$ and 8 am on the weekdays and between $6 \mathrm{pm}$ and 9 am during the weekend.

\section{Staffing}

Intensivists are present during the day time and make rounds at the bedside at least twice a day. Each day a multidisciplinary meeting is held in which all patients are discussed. During off hours, an intensivist guard is available for consultation 24 hours a day, seven days a week, and has no other responsibilities apart from ICU related patient care. The nurse to patient ratio is about 1:2 during the day time and during the night time. Data are collected prospectively for each patient. Demographic, clinic, acute physiology and chronic health evaluation (APACHE II) score, ${ }^{6}$ time of admission, length of stay (LOS), and ICU mortality are among the data collected. The statistical data were collected prospectively from ICU databases and analyzed with SPSS version 10 (IBM Corporation, Armonk, NY, USA). Symmetrical variables were reported as the mean \pm standard deviation and the means were compared using Student's $t$-test. Asymmetrical data were reported as median and interquartile range (IQR). Medians were compared using the Mann-Whitney test. Differences in proportions were compared using chi-square tests. Odds ratios and confidence intervals were also reported. $P<0.05$ was considered statistically significant. Logistic regression analysis was used to identify risk factors associated with ICU mortality at various days and times of admission.

\section{Results}

A total of 195 patients were included in the study; 125 (63.6\%) were admitted during the day time and 70 (36.4\%) were admitted during off hours. The median age was 32 years (23-59 years). The sex ratio was 1 female: 2 males and the mean APACHE II score was $14.7 \pm 3$. Three quarters of the patients were admitted from the emergency department. The rest were admitted from other services of medicine and surgery from the same hospital and $38 \%$ of patients had a medical history. With regards to the reason for admission, $38 \%$ of patients were admitted for traumatism and 29\% for surgery, in particular neurosurgery, gastrointestinal surgery, and trauma surgery. Also, $33.6 \%$ of patients had medical pathology and $64 \%$ of patients had mechanical ventilation. The median ICU LOS was 5 days (3-10 days). ICU mortality was $43.6 \%$.

Baseline characteristics were not equal for the group of patients admitted during the day time as compared with those admitted during off hours (Table 1). The difference was most marked for the sex of patients and pathology reason for admission, most of the patients admitted during the off hours were male $(75 \%$ versus $58 \%, P=0.01)$. More patients were admitted after traumatism during the off hours than during the day time ( $64 \%$ versus $24 \%, P=0.001$ ). There was no significant difference in ICU mortality for time of ICU admission ( $51 \%$ versus $37 \%$, day time versus night time, respectively, $P=0.05$ ). In univariate and multivariate analyses, and controlling for factors associated with mortality, such as age, sex, APACHE II, LOS, trauma, and time of admission, no statistically significant difference in ICU mortality was found with time of admission. Only mechanical ventilation was a significant predictive factor for intensive care mortality (Table 2).

\section{Discussion}

Our study demonstrates that the demographic characteristics were similar for patients admitted during the day time as compared with those admitted during off hours, except for the frequency of male patients and higher trauma during off hours. It is not surprising that a significantly larger number of patients who are admitted at night or during weekends are made up of trauma patients because of the frequency of road accidents in our region and risk behaviors among young males. Although nights and weekends are frequently considered the off hours, with lower staffing levels and availability of services or resources, there are higher risks of mortality and poor outcome. We found no significant differences in ICU mortality and LOS associated with the day and time of ICU admission. There are several possible explanations for our finding. The differences in institutional practice in organizational aspects of care may be responsible. Although our findings show no difference in mortality between patients admitted on day time hours compared to off hours, it was found that mortality remains high whatever the time of admission. We can explain this by the initial severity of the patients at admission and the selection of the most severe patients because our department is the only adult ICU in the region and has a limited capacity. Also, the frequency of traumatic 
Table I Comparison of clinical and epidemiological characteristics of the group of patients admitted during the day time versus the group admitted during off hours in intensive care unit

\begin{tabular}{|c|c|c|c|c|}
\hline \multirow[t]{2}{*}{ Variables } & \multirow[t]{2}{*}{ All patients } & \multicolumn{2}{|l|}{ Group } & \multirow[t]{2}{*}{$P$-value ${ }^{a}$} \\
\hline & & Day time & Off hours & \\
\hline Total patient numbers & 195 & 125 & 70 & \\
\hline Sex, male/female, (\% male) & $130 / 65(66)$ & $60 / 43(58)$ & 68/22 (75) & 0.01 \\
\hline Age, median (IQR), years & $32(23-52)$ & $30(22,54)$ & $35(23,52)$ & 0.9 \\
\hline APACHE II score at admission (mean \pm SD) & $14.7 \pm 3$ & $14.9 \pm 5.6$ & $15 \pm 6.4$ & 0.4 \\
\hline Presence of medical history & $74(38)$ & $41(34)$ & $33(45)$ & 0.29 \\
\hline Cardiovascular & $13(6.6)$ & $9(7)$ & $4(5.7)$ & 0.06 \\
\hline Diabetes & $15(7.7)$ & $10(8)$ & $5(7)$ & 0.15 \\
\hline Respiratory & II (5.6) & $5(4)$ & $6(8.6)$ & 0.3 \\
\hline Neurological & $14(7.2)$ & $8(8)$ & $6(8.6)$ & 0.25 \\
\hline Others & $21(10.8)$ & $9(7)$ & $12(17)$ & 0.06 \\
\hline \multicolumn{5}{|l|}{ Admissions type } \\
\hline Trauma & $75(38)$ & $30(24)$ & $45(64)$ & $<0.001$ \\
\hline Medical pathology & $63(33.6)$ & $31(25)$ & $32(45)$ & 0.09 \\
\hline Sepsis & II (5.7) & $5(4.9)$ & $6(6.5)$ & 0.1 \\
\hline Respiratory distress & II (5.7) & $6(5.8)$ & $5(5.6)$ & 0.9 \\
\hline Shock & $10(5.2)$ & $4(3.9)$ & $6(6.5)$ & 0.3 \\
\hline Metabolic & $13(6.7)$ & $6(5.8)$ & $7(7.8)$ & 0.6 \\
\hline Neurologic & $8(4)$ & $5(4.9)$ & $3(3.4)$ & 0.8 \\
\hline Intoxication & $10(5.2)$ & $5(4.9)$ & $5(5.6)$ & 0.7 \\
\hline Surgery & $57(29)$ & $42(60)$ & $14(20)$ & 0.06 \\
\hline Mechanical ventilation & $126(64)$ & $65(63)$ & $61(65)$ & 0.7 \\
\hline Median ICU LOS (IQR) & $5(3$ tol0) & $5(3$ to 10$)$ & 7 (4 to I3) & 0.07 \\
\hline ICU mortality & $85(43)$ & $38(37)$ & $47(5 \mathrm{I})$ & 0.05 \\
\hline
\end{tabular}

Notes: alpha risk of first species. Unless otherwise specified the data are presented as number, followed by percentage in brackets.

Abbreviations: APACHE, acute physiology and chronic health evaluation score; ICU, intensive care unit; IQR, interquartile range; LOS, length of stay; SD, standard deviation.

pathology related to the road accidents is a real health problem in our context. This has allowed us to take some measures for the prevention of road accidents through outreach of the media and the general public, and especially to intensify the initial management of patients before admission to intensive care by the most experienced emergency staff.

Our study has limitations concerning the small sample of patients and the study was carried out in only one center which receives a lot of young male trauma patients, who are

Table 2 Risk factors for patient mortality

\begin{tabular}{|c|c|c|c|}
\hline Parameters in the model & Wald & $P$-value ${ }^{a}$ & Adjusted OR $(95 \% \mathrm{Cl})$ \\
\hline \multicolumn{4}{|l|}{ Logistic regression model } \\
\hline Age (years) & 0.95 & 0.3 & I $(0.97$ to I) \\
\hline Sex (male) & 0.17 & 0.68 & I.I 17 (0.54 to 2.57$)$ \\
\hline Presence of medical history & 0.45 & 0.50 & 0.75 (0.33 to I.7) \\
\hline APACHE II score & 2.17 & 0.14 & 0.9 (0.9 to I) \\
\hline Trauma admission & 0.08 & 0.09 & $0.46(0.19$ to 1.12$)$ \\
\hline Mechanical ventilation & 12 & 0.001 & 4.17 ( 1.86 to 9.37$)$ \\
\hline Length of stay & 0.009 & 0.92 & I $(0.96$ to I.04) \\
\hline Off hours admission & 0.89 & 0.34 & $1.38(0.70$ to $2.7 \mathrm{I})$ \\
\hline
\end{tabular}

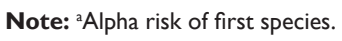

Abbreviations: APACHE, acute physiology and chronic health evaluation score; OR, odds ratio. generally accepted at night. This is due to decreased vigilance, risky behaviors, and the bad condition of roads leading to a considerable increase in traffic accidents. In our analysis we have not taken into account holidays or vacations that may significantly affect the result. Significant heterogeneity in results has been found in several large studies that evaluated night time admission in ICU, all in retrospective cohorts and all defined off hours differently. ${ }^{7-11}$ Uusaro et al have shown increased mortality of patients admitted in 80 ICUs during weekends when compared to weekdays. ${ }^{5}$ Another multicenter retrospective study carried out in Canada during 2008 by Laupland et al has shown increased mortality for patients admitted at night compared to those admitted during the day but without increasing mortality during the weekend. ${ }^{9}$ Other studies have shown conflicting results. ${ }^{12-14}$ The study by Luyt et al, conducted in 23 ICUs over 3 years in 2007, did not show a significant difference in the mortality for patients admitted during off hours when compared to those admitted during the day time. ${ }^{7}$ Differences in mortality can be explained by differences in disease severity and differences in the definitions of day and hours of admission may also explain the difference in results between studies. A recent meta-analysis 
has studied this problem ${ }^{1}$ and it included ten cohort studies; eight evaluated night admissions and six studies evaluated weekend admission that demonstrated that a night admission was not associated with an increased mortality. However, patients admitted over the weekend have a significant increase in the risk of death. This meta-analysis has a number of limitations, most importantly, different definitions were used for both night time and weekend hours, the description of the organization was not well described in all studies, and different severity adjustment tools were used.

\section{Conclusion}

Our study found no difference in mortality whatever the time of admission, with the same staff and the same organization. We can conclude that off hours care is not necessarily inadequate. For ICU managers, it is important to know how to maintain adequate quality of care around the clock. However, mortality is high no matter the time of admission and this is due to the selection of severely ill patients, especially victims of road accidents. This should encourage us to develop more mobile emergency care, more experienced staff in the emergency department, and in particular, raise awareness of public and political leaders to prevent traffic accidents.

\section{Disclosure}

The authors report no conflict of interest in this work.

\section{References}

1. Cavallazzi R, Marik PE, Hirani A, Pachinburavan M, Vasu TS, Leiby BE. Association between time of admission to the ICU and mortality: a systematic review and meta-analysis. Chest. 2010;138(1):68-75.
2. Rivers E, Nguyen B, Havstad S, et al. Early Goal-Directed Therapy Collaborative Group. Early goal-directed therapy in the treatment of severe sepsis and septic shock. N Engl J Med. 2001;345(19): $1368-1377$.

3. Arabi Y, Alshimemeri A, Taher S. Weekend and weeknight admissions have the same outcome of weekday admissions to an intensive care unit with onsite intensive coverage. Crit Care Med. 2006;34(3):605-611.

4. Morales IJ, Peters SG, Afessa B. Hospital mortality rate and length of stay in patients admitted at night to the intensive care unit. Crit Care Med. 2003;31(3):858-863.

5. Uusaro A, Kari K, Ruokonen E. The effects of ICU admission and discharge times on mortality in Finland. Intensive Care Med. 2003;29(12):2144-2148.

6. Knaus WA, Draper EA, Wagner DP, Zimmerman JE. APACHE II: a severity of disease classification system. Crit Care Med. 1985;13(10):818-829.

6. Luyt CE, Combes A, Aegerter P, et al. Mortality among patients admitted to intensive care units during weekday shifts compared with off hours. Crit Care Med. 2007;35(1):3-11.

7. Wunsch H, Mapstone J, Brady T, Hanks R, Rowan K. Hospital mortality associated with day and time of admission to intensive care units. Intensive Care Med. 2004;30(5):895-901.

8. Laupland KB, Shahpori R, Kirkpatrick AW, Stelfox HT. Hospital mortality among adults admitted to and discharged from intensive care on weekends and evenings. J Crit Care. 2008;23(3):317-324.

9. Sheu CC, Tsai JR, Hung JY, et al. Admission time and outcomes of patients in a medical intensive care unit. Kaohsiung J Med Sci. 2007;23(8):395-404.

10. Meynaar IA, van der Spoel JI, Rommes JH, van Spreuwel-Verheijen M, Bosman RJ, Sprong PE. Off hours admission to an intensivist-led ICU is not associated with increased mortality. Crit Care. 2009;13(3):R84.

11. Lee KK, Ng I, Ang BT. Outcome of severe head injured patients admitted to intensive care during weekday shifts compared to nights and weekends. Ann Acad Med Singapore. 2008;37(5):390-396.

12. Barnett MJ, Kaboli PJ, Sirio CA, Rosenthal GE. Day of the week of intensive care admission and patients outcomes: a multisite regional evaluation. Med Care. 2002;40(6):530-539.

13. Ensminger SA, Morales IJ, Peters SG, et al. The hospital mortality of patients admitted to the ICU on weekends. Chest. 2004;126(4): $1292-1298$.
International Journal of General Medicine

\section{Publish your work in this journal}

The International Journal of General Medicine is an international, peer-reviewed open-access journal that focuses on general and internal medicine, pathogenesis, epidemiology, diagnosis, monitoring and treatment protocols. The journal is characterized by the rapid reporting of reviews, original research and clinical studies across all disease areas.

\section{Dovepress}

A key focus is the elucidation of disease processes and management protocols resulting in improved outcomes for the patient.The manuscript management system is completely online and includes a very quick and fair peer-review system. Visit http://www.dovepress.com/ testimonials.php to read real quotes from published authors. 